\title{
The design of autonomous DNA nano-mechanical devices: Walking and rolling DNA
}

\author{
JOHN H. REIF \\ Department of Computer Science, Duke University, Box 90129, Durham, NC 27708-0129, \\ USA (E-mail: reif@cs.duke.edu)
}

\begin{abstract}
We provide designs for the first autonomous DNA nanomechanical devices that execute cycles of motion without external environmental changes. These DNA devices translate along a circular strand of ssDNA and rotate simultaneously. The designs use various energy sources to fuel the movements, include (i) ATP consumption by DNA ligase in conjunction with restriction enzyme operations, (ii) DNA hybridization energy in trapped states, and (iii) kinetic (heat) energy. We show that each of these energy sources can be used to fueI random bidirectional movements that acquire after $n$ steps an expected translational deviation of $O(\sqrt{n})$. For the devices using the first two fuel sources, the rate of stepping is accelerated over the rate of random drift due to kinetic (heat) energy. Our first DNA device, which we call walking DNA, achieves random bidirectional motion around a circular ssDNA strand by use of DNA ligase and two restriction enzymes. Our other DNA device, which we call rolling $D N A$, achieves random bidirectional motion without use of DNA ligase or any restriction enzyme, and instead using hybridization energy. We also describe how to modify the design for the rolling DNA device to include a "latching mechanism" that fixes the wheels position at specified location along the road, so as to provide for overall unidirectional translational movement.
\end{abstract}

Key words: DNA, molecular, motor nanomechanical device, nạostructure

\section{Introduction}

Prior work. Nanomechanical devices built of DNA have been developed using two distinct approaches: (i) Seeman's group (Musser, 1999) used rotational transitions of dsDNA conformations between the B-form (right handed) to the Z-form (left-handed) controlled by ionic effector molecules and extended this technique to be DNA sequence dependant (Yan, Zhang, Shen and Seeman, 2002). (ii) Yurke and Turberfield (Yurke, Turberfield, Mills, Simmel and Neumann, 2000) demonstrated a series of DNA nanomechanical devices that used a fuel DNA acting as a hybridization catalyst to generate a sequence of motions in another strand of DNA (called DNA tweezers); the two strands of DNA bind and unbind with the overhangs to alternately open and shut the tweezers.

\section{Preprint of paper appearing in natural Computing, DAas special issue, vol. 2, p 439-461, (2003).}


These prior DNA nanomechanical devices have some key restrictions that restrict their usefulness:

(a) they can only execute one type of motion (rotational or translational). It seems feasible to re-design these DNA nanomechanical devices to have both translational and rotational motion, but this still needs to be done (experimentally tested).

(b) More importantly, these prior DNA devices do not run autonomously: to make repeated motions they either require environmental changes such as temperature cycling, or bead treatment of biotin-streptavidin beads, or require feeding more or different fuel strands to cycle the device.

The technical challenge. A key challenge, which we address in this paper, is to make an autonomous DNA nanomechanical device that executes cycles of motion (either rotational or translational or both) without external environmental changes.

Our results. We provide designs for two autonomous DNA nanomechanical devices. Both move along a circular ssDNA strand which we call a road and simultaneously exhibit both translational and rotational movement. However, they use distinct energy sources to fuel the movements.

(i) Our first nanomechanical device design, which we call walking DNA and illustrate in Figure 1a, uses as its energy source ATP consumption by DNA ligase in conjunction with two restriction enzyme operations. Our walking DNA construction (though technically quite distinct and with a different objective) might be viewed as analogous to Shapiro's recent autonomous DNA computing machine (Benenson, Paz-Elizur, Adar, Keinan, Livneh and Shapiro, 2001).

(ii) Our next nanomechanical device design, which we call rolling DNA and illustrate in Figure 1b,makes no use of DNA ligase or any restriction enzyme. Instead, it uses as its energy source the hybridization energy of DNA in temporally trapped states. This involves the use of fuel DNA and the application of DNA catalyst techniques for liberating DNA from these loops conformations, and to harness their energy as they transit into lower energy conformations. Unlike the prior work of (Yurke, Turberfield, Mills, Simmel and Neumann, 2000), our resulting nanomechanical device is autonomous. We also observe that a similar movement, but possibly at an unusable lower rate, can also be achieved using this basic design using simply kinetic (heat) energy.

Potential applications. In principle, these DNA nanomechanical devices can be incorporated at multiple sites of larger DNA nanostructures such as selfassembled DNA lattices. The DNA nanomechanical devices might be used to 


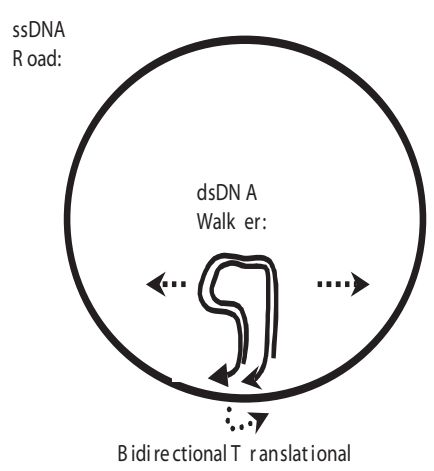

\& Rotational M ovement

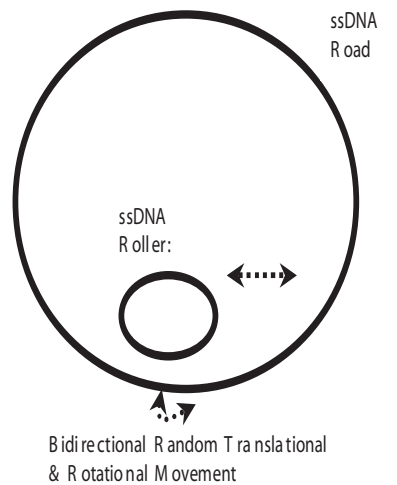

\& Rotational Movement

Figure 1. (a)The DNA Walking Mechanism. (b)The DNA Rolling Mechanism.

induce conformational changes that store state information and to sequence between distinct conformations. Possible applications include: (a) Array Automata: The state information could be stored at each site of a regular DNA lattice (see Winfree, Wenzler and Seeman, 1998; Seeman, 1999; LaBean, Yan, Kopatsch, Liu, Winfree, Reif and Seeman, 2000; LaBean, Winfree and Reif, 2000; Mao, LaBean, Reif and Seeman, 2000; Reif, LaBean and Seeman, 2001; Reif, 2001), and additional mechanisms for finite state transiting would provide for the capability of a cellular array automata. (b) Nanofabrication: These capabilities might be used to selectively control nanofabrication stages. The size or shape of a DNA lattice may be programmed through the control of such sequence-dependent devices and this might be used to execute a series of foldings (similar to Origami paper folding techniques) of the DNA lattice to form a variety of $3 \mathrm{D}$ conformations and geometries.

\section{Our walking DNA device}

Here we describe a DNA nanomechanical device (see Figure 1a) that achieves autonomous bidirectional translational and rotational motion along a DNA strand. We will be using, as the energy source, ATP consumption by DNA ligase in conjunction with restriction enzyme operations.

Complementarity and reversal notation. We let ssDNA denote singly stranded DNA and let dsDNA denote doubly stranded DNA.

We will use superscript $\mathrm{R}$ to denote the reverse of a sequence. Given a sequence of oglionucleotides $A_{0}, A_{0}, \ldots, A_{n-1}, A_{n}$ (with reverses respectively denoted as $A_{0}{ }^{R}, A_{1}{ }^{R}, \ldots, A_{n-1}{ }^{R}, A_{n}{ }^{R}$ ), the reverse of $A_{0} A_{0} \ldots$ $A_{n-1} A_{n}$ is the sequence $\left(A_{0} A_{1} \ldots A_{n-1} A_{n}\right)^{R}=A_{n}{ }^{R} A_{n-1}{ }^{R} \ldots A_{1}{ }^{R} A_{0}{ }^{R}$. 


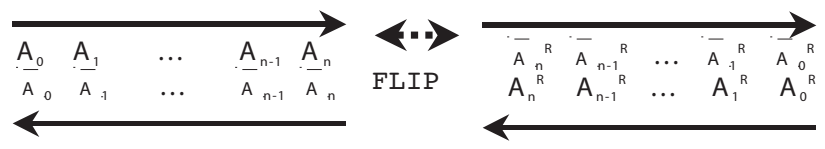

Figure 2. A dsDNA sequence and its flip.

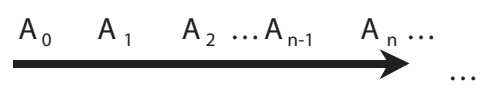

Figure 3. The DNA Road.

We will also use overbar to denote the complement of an ssDNA sequence. Note that base pairing among the DNA bases A, G, C, T provides $\bar{A}=T, \bar{T}=A, \bar{C}=T$, and $\bar{T}=C$. Given a sequence of oglionucleotides $A_{0}, A_{0}, \ldots, A_{n-1}, A_{n}$ (with complements respectively denoted as $\overline{A_{0}}, \overline{A_{1}}, \ldots, \overline{A_{n-1}}, \overline{A_{n}}$ ) then we will define the complement of $A_{0} A_{0} \ldots A_{n-1} A_{n}$ to be the sequence $\overline{A_{0} A_{1} \ldots A_{n-1} A_{n}}=$ $\overline{A_{n}} \overline{A_{n-1}} \ldots \overline{A_{1}} \overline{A_{0}}$ with the individual complemented oglionucleotides listed in reverse order.

The upper portion of Figure 2 illustrates the dsDNA resulting from hybridization of an upper ssDNA sequence $A_{0} A_{1} \ldots A_{n-1} A_{n}$ (written in $5^{\prime}$ to $3^{\prime}$ direction from left to right) with an lower ssDNA sequence $\overline{A_{n}} \overline{A_{n-1}} \ldots \overline{A_{1}} \overline{A_{0}}$ (with the symbols written in $3^{\prime}$ to $5^{\prime}$ direction from left to right). The right portion of Figure 2 illustrates the effect on this notation when we flip over (both vertically and horizontally) the dsDNA sequence, resulting in an essentially identical dsDNA sequence but with the component sequences reversed: with a lower sequence $A_{n}^{R} A_{n-1}^{R} \ldots A_{1}^{R} A_{0}^{R}$ (written in $3^{\prime}$ to $5^{\prime}$ direction from left to right) and an upper ssDNA sequence ${\overline{A_{n}}}^{R}{\overline{A_{n-1}}}^{R} \ldots{\overline{A_{1}}}^{R}{\overline{A_{0}}}^{R}$ (with the symbols written in $5^{\prime}$ to $3^{\prime}$ direction from left to right).

Overview of our walking DNA device construction. The basic idea is to form a circular repeating strand $\mathrm{R}$ of ssDNA we call the road, which will be written in $5^{\prime}$ to $3^{\prime}$ direction from left to right. The road (See Figure 3 ) will consist of an even number $\mathrm{n}$ of subsequences, which we call steppingstones, indexed from 0 to $\mathrm{n}-1$ modulo $\mathrm{n}$. The $i^{t h}$ steppingstone consists of a length $\mathrm{L}$ (where $\mathrm{L}$ is between 15 to 20 base pairs) sequence $A_{i}$ of ssDNA. (In our constructions, the $A_{i}$ repeat with a period of 2.)

Let us designate the direction $5^{\prime}$ to $3^{\prime}$ to be direction to the right on the road $\mathrm{R}$, and let us designate the direction $3^{\prime}$ to $5^{\prime}$ to be direction to the left on the road R. At any given time, there is a unique partial duplex DNA strand $W_{i}$ which we call the $i^{\text {th }}$ walker with $3^{\prime}$ ends $\bar{A}_{\mathbf{i}-1}$ and $\bar{A}_{\mathbf{i}}$ that are hybridized 


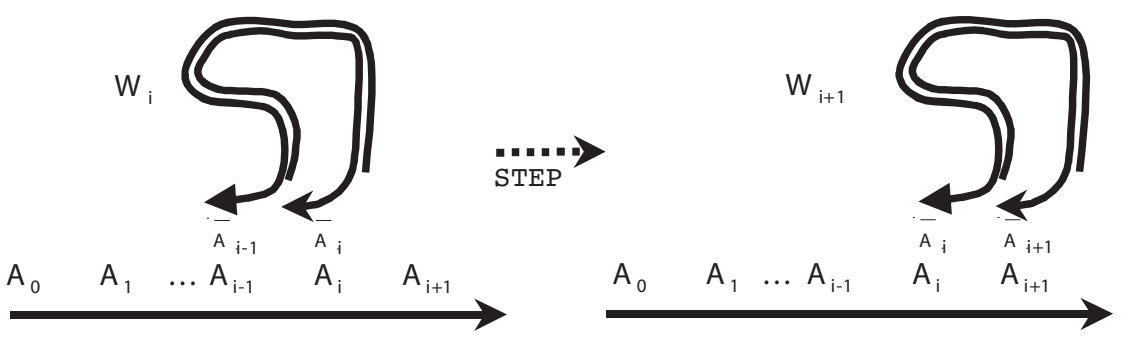

Figure 4. A step of the DNA walker.

to consecutive $(i-1)^{t h}$ and $i^{\text {th }}$ steppingstones $A_{i-1}$ and $A_{i}$, respectively, as illustrated on the left portion of Figure 4.

The goal of the device construction. The below construction will have the goal of getting the $i^{\text {th }}$ walker $W_{i}$ to reform to another partial duplex DNA strand, namely the $(i+1)^{\text {th }}$ walker $W_{i+1}$ which is shifted one unit over to the left or the right. In the case of the latter movement to the right, the movement results in the $3^{\prime}$ ends $\bar{A}_{\mathbf{i}}$ and $\bar{A}_{\mathbf{I}+\mathbf{1}}$ being hybridized to consecutive $i^{t h}$ and $(i+1)^{t h}$ steppingstones $A_{i}$ and $A_{i+1}$, respectively, as illustrated on the right portion of Figure 4 (the movement to the left is reversed). Hence, the movement we will achieve is bidirectional, translational movement either in the $5^{\prime}$ to $3^{\prime}$ direction (from left to right) on the road, or in the $3^{\prime}$ to $5^{\prime}$ direction. We will describe in detail the movement in the $5^{\prime}$ to $3^{\prime}$ direction, and then observe that movement in the $3^{\prime}$ to $5^{\prime}$ direction is also feasible. We cycle back in 2 stages, so that $W_{i+2}=W_{i}$ for each stage $i$. To achieve the movements, we will use two distinct type-2 restriction enzymes. We will also make use of DNA ligase, which provides a source of energy (though ATP consumption) and a high degree of irreversibility.

Simultaneous translational and rotational movements. Due to the well known secondary structure of B-form dsDNA (rotating $2 \pi$ radians every approx 10.5 bases), it follows that in each such step of translational movement, the walker will rotate around the axis of the road by approximately $L 2 \pi / 10.5=$ $L \pi / 5.25$ radians. Hence the DNA walker construction will execute a very simple type of motion with simultaneous translation and rotation.

See the Discussion Section for a description of the use of various methods (loosely immobilizing the road and suggested settings of road and walker concentrations) to avoid unwanted interactions.

Details of our device construction. In the following detailed description given below, we describe the sequence of movements involving all the components: 
(b) The DNA stepper $S_{i}$ :

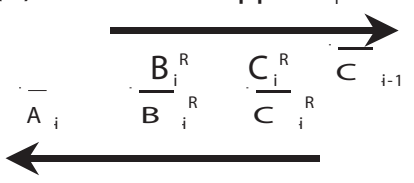

(a) The DNA walker $W_{i}$ :

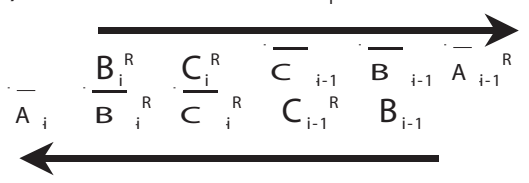

Figure 5. (a)The DNA walker $W_{i}$. (b)The DNA stepper $S_{i}$.

walker, road, restriction enzymes and ligase. And we provide a sequence of cartoon Figures illustrating these movements.

Definition of the walker and oglionucleotides used in walking DNA construction. For each $\mathrm{i}=0,1$, we define ssDNA (in the $5^{\prime}$ to $3^{\prime}$ direction): (a) $\mathrm{G}_{i}$ $=B_{i}^{R} C_{i}^{R} \bar{C}_{i-1} \bar{B}_{i-1} \bar{A}_{i-1}^{R}$ and (b) $H_{i}=B_{i-1}^{R} C_{i-1}^{R} \bar{C}_{i} \bar{B}_{i} \bar{A}_{i}{ }^{R}$. Here $B_{i} C_{i}$ and the previously defined $A_{i}$ are distinct oglionucleotides of low annealing cross-affinity. To ensure we cycle back in 2 stages, the subscripts of the $A_{i} B_{i}$ $C_{i}$ are taken modulo 2, and so: $A_{i+1}=A_{i-1}, B_{i+1}=B_{i-1}$ and $C_{i+1}=C_{i-1}$ for each i. Note that $\mathrm{G}_{i}$ has as a subsequence $B_{i}^{R} C_{i}^{R} \bar{C}_{i-1} \bar{B}_{i-1}$ and $H_{i}$ has as a subsequence, the reverse complement $B_{i-1}^{R} C_{i-1}^{R} \bar{C}_{i} \bar{B}_{i}$ of that subsequence $B_{i}^{R} C_{i}^{R} \bar{C}_{i-1} \bar{B}_{i-1}$ found within $\mathrm{G}_{i}$. We define the $i^{\text {th }}$ walker $W_{i}$ to be the partial duplex resulting by hybridization of $\mathrm{G}_{i}$ and $H_{i}$ at these complementary subsequences, with ssDNA $3^{\prime}$ overhangs $\bar{A}_{i-1}$ and $\bar{A}_{i}$, as given in Figure 5a.

We inductively assume that $i^{\text {th }}$ walker $W_{i}$ has the $3^{\prime}$ end $\bar{A}_{i-1}$ of its component strand $\mathrm{G}_{i}$ hybridized to steppingstone $A_{i-1}$ on the road $\mathrm{R}$ and the $3^{\prime}$ end $\bar{A}_{i}$ of its component strand $H_{i}$ hybridized to steppingstone $A_{i}$ on the road $\mathrm{R}$.

Definition of the stepper. To aid us in this movement, for each $\mathrm{i}=0,1$ we include in solution the following partial duplex $S_{i}$ which we call the $i^{\text {th }}$ stepper strand illustrated in Figure 5b.

Note that each of the $i^{\text {th }}$ steppingstone $A_{i}$ subsequences will hybridize with a complementary subsequence $\bar{A}_{i}$ of the $i^{\text {th }}$ stepper $S_{i}$. We assume that this occurs at each steppingstone, except the steppingstones where the walker's ends are hybridized, as illustrated on the left portion of Figure 9.

Restriction enzyme cleavage of the walker. For each $\mathrm{i}=0$, 1 , we use a type two restriction enzyme that matches with the duplex subsequence containing $C_{i-1} B_{i-1}$ and its complement $\bar{C}_{i-1} \bar{B}_{i-1}$ within $W_{i}$, and then cleaves $W_{i}$ just before $\bar{C}_{i}$ and just after $C_{i}$, as illustrated on the right portion of Figure 6. This can result in the following products: 


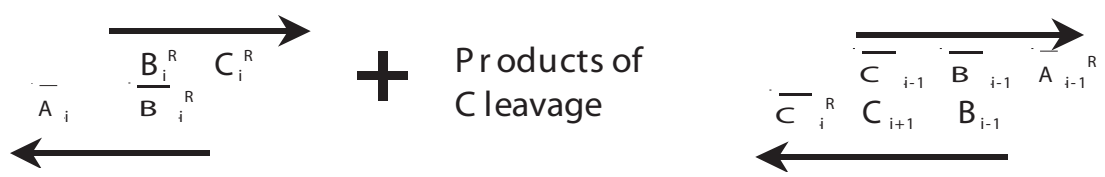

Figure 6. Products of the cleavage of the DNA walker.

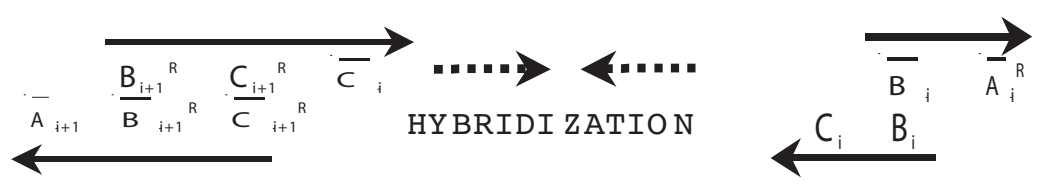

Figure 7. Hybridization with the DNA walker.

(P1) A $i^{\text {th }}$ truncated walker $T W_{i}$ (still attached to the $i^{\text {th }}$ steppingstone $S_{i}$ ) consisting of a partial duplex with an ssDNA overhang $\left(C_{i}\right)^{R}$ at one $3^{\prime}$ end, as illustrated on the left portion of Figure 6, and also

(P2) A partial duplex, as also illustrated on the right portion of Figure 6, which we can observe is the $(i-1)^{t h}$ stepper $S_{i-1}$, still attached to the $(i-1)^{\text {th }}$ steppingstone $S_{i-1}$ with an ssDNA overhang $\left(\bar{C}_{i}\right)^{R}$ at one $3^{\prime}$ end. To see this is the $(i-1)^{t h}$ stepper $S_{i-1}$, note that the right portion of Figure 6 can be flipped left to right and top to bottom so as to be equivalently viewed (as illustrated on the left portion of Figure 7) as $\bar{C}_{i}$; since $\bar{C}_{i}=\bar{C}_{i-2}$, this above cleavage product is identical to above defined $(i-1)^{t h}$ stepper $S_{i-1}$. (See an example of a flip in Figure 2. Also note that the left portion of Figure 6 can be flipped left to right and top to bottom so as to be equivalently viewed as illustrated on the right portion of Figure 7.)

We assume that the sequences of sufficiently short length that the only strands with an ssDNA subsequence $\bar{C}_{i}$ which can possibly hybridize with the ssDNA subsequence $C_{i}$ of the above $i^{t h}$ truncated walker $T W_{i}$ are the adjacent steppers $S_{i-1}$ and $S_{i+1}$.

Reversals. It will be important to observe, when at the end of this section further below we observe bi-directionality of the movement of the walker, that the walker has two possible (dual) restriction enzyme recognition sites, and so the dual use of the two restriction enzymes also can result in other pair of products, resulting in a reversal of movement. However, to simplify the discussion, we ignore these reversals for the moment.

Stalls. Observe that it is certainly quite possible that the above cleavage operation might simply be reversed by re-hybridization of subsequence 


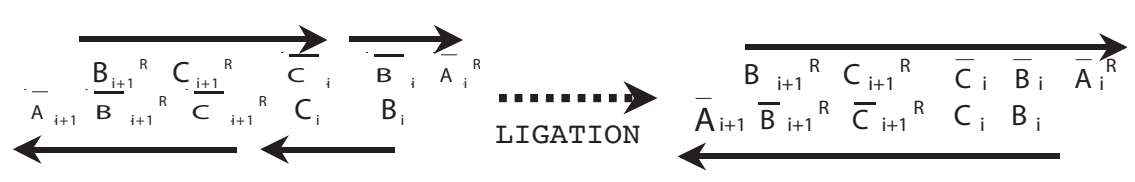

Figure 8. Ligation within the DNA walker.

$\bar{C}_{i}$ of stepper $S_{i-1}$ with the complementary subsequence $C_{i}$ of the above truncated walker $T W_{i}$ (and possibly a subsequent ligation), reforming the original walker $W_{i}$. We will call this restriction enzyme cutting and then rehybridization a stall event for $W_{i}$. Each stall event has a constant probability $q$, where $0<q<1$. The consecutive repetition of stall events for $W_{i}$ might continue any number of times, but as we shall see, the likelihood drops rapidly with the number of repeated stall events. To see this, observe that the number of repeats has, by definition, a geometric probability distribution with geometric parameter $p=1-q$. This geometric probability distribution is known to have expectation $E=p /(1-p)$, which is a fixed constant. Furthermore, the probability of $s E$ repeats of consecutive stall events for $W_{i}$ (that is, a factor s more than the expected number $E$ ) can be shown to drop exponentially with $s$, for $s>2$.

The stepping of the walker. In the following, we now consider the case where there has already been a number of stall events for $W_{i}$, but now there is not a further repeat of a stall event for $W_{i}$. In this case, we show that what eventually occurs is the intended modification of the truncated walker $T W_{i}$ into the $(i+1)^{t h}$ walker $W_{i+1}$. Observe that by flipping the left portion of Figure 6 for $\mathrm{TW}_{i}$ (the flip is top to bottom and left to right), the truncated walker $\mathrm{TD}_{i}$ can be redrawn as illustrated on the right portion of Figure 7 and the $(i+1)^{t h}$ stepper strand $S_{i+1}$ is by definition as illustrated on the left portion of Figure 7.

This $(i+1)^{t h}$ stepper strand $S_{i+1}$ was assumed to be hybridized with a complementary subsequence $\bar{A}_{i+1}$ of $(i+1)^{t h}$ stepper $S_{i+1}$. This $(i+1)^{t h}$ stepper strand $S_{i+1}$ can also hybridize with the truncated walker $T W_{i}$ at their $\bar{C}_{i}$ and $C_{i}$ ssDNA overhangs, to form the duplex as illustrated on the left portion of Figure 8, and the DNA ligase can concatenate the strands, forming the following duplex as illustrated on the right portion of Figure 8.

But the above duplex is by definition the $(i+1)^{t h}$ walker $W_{i+1}$. We now claim that this $(i+1)^{t h}$ walker $W_{i+1}$ has its $3^{\prime}$ ends hybridized to consecutive steppingstones $A_{i}$ and $A_{i+1}$ in its required position as given on the right portion of Figure 9. This follows since:

(a) A $3^{\prime}$ end of the $i^{t h}$ walker $W_{i}$ was assumed to be hybridized with a complementary subsequence $\bar{A}_{i}$ of stepper $S_{i}$, so that the same $3^{\prime}$ end of 


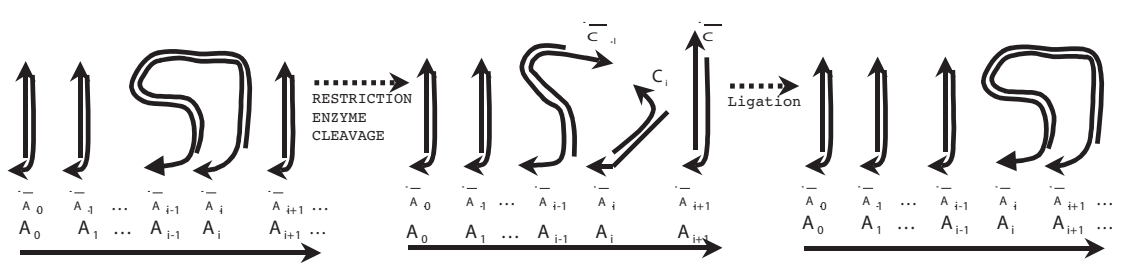

Figure 9. A step of the DNA walker.

the $i^{t h}$ truncated walker $T W_{i}$ remains hybridized with a complementary subsequence $\bar{A}_{i}$ of stepper $S_{i}$, and further the $(i+1)^{t h}$ walker $W_{i+1}$ inherits this hybridization of that $3^{\prime}$ end.

(b) A $3^{\prime}$ end of the $(i+1)^{t h}$ steppingstone subsequence $A_{i+1}$ was assumed to be hybridized with a complementary subsequence $\bar{A}_{i+1}$ of stepper $S_{i+1}$, so it follows the $(i+1)^{\text {th }}$ walker $W_{i+1}$ inherits this hybridization of that $3^{\prime}$ end.

The above described movement of the $i^{\text {th }}$ walker $W_{i}$ to reformed $(i+1)^{t h}$ walker $W_{i+1}$ on the road $\mathrm{R}$ is always from direction $3^{\prime}$ to $5^{\prime}$ once ligation occurs, and it is very unlikely to be reversed after that. The above discussion of the feasibility of movement in the $3^{\prime}$ direction can be summarized as follows: We assumed the situation STATE(i) where:

(a) walker $W_{i}$ has the $3^{\prime}$ end of $\mathrm{G}_{i}$ hybridized to steppingstone $A_{i-1}$ and the $3^{\prime}$ end of $H_{i}$ hybridized to steppingstone $A_{i}$, and also

(b) the steppingstone $A_{k}$ subsequence is hybridized with a complementary subsequence $\bar{A}_{k}$ of stepper $S_{k}$, for each $\mathrm{k}$, where $k<i-1$ ork $>i$.

The above discussion has demonstrated that given STATE(i), and then in an expected constant number of phases, it is feasible for us to have a movement in the $3^{\prime}$ direction on the road to STATE(i+1). But this does not complete our description of all possible movements of the walking DNA construction.

Feasible reverse movements. The above does not ensure the movement is only in the $5^{\prime}$ direction along the road. In the above subsection titled "Restriction Enzyme Cleavage of the Walker" we described a pair of products (P1) and (P2) that can result from the use of the restriction enzymes described there. (To simplify the description of $3^{\prime}$ movements, we temporarily ignored other possible products in that above discussion.) We now observe that the walker actually has two possible (dual) restriction enzyme recognition sites, so dual use of the two restriction enzymes defined there also can result in:

$\left(\mathrm{P}^{\prime}\right)$ A partial duplex, consisting of the $i^{\text {th }}$ stepper $S_{i}$ (still attached to the $i^{\text {th }}$ steppingstone $S_{i}$ ) with an ssDNA overhang $\left(C_{i-1}\right)^{R}$ at one $3^{\prime}$ end, or 
(P2') A $(i-1)^{\text {th }}$ truncated walker $T W_{i-1}$ (still attached to the $(i-1)^{\text {th }}$ steppingstone $S_{i-1}$ ) consisting of a partial duplex with an ssDNA overhang $\left(\bar{C}_{i-1}\right)^{R}$ at one $3^{\prime}$ end.

By a similar argument to the above, it can easily be shown: there is also a feasible movement of the walker in the $5^{\prime}$ direction on the road from STATE(i) to STATE(i-1). By symmetry, both these possible movements have equal likelihood. Summarizing, we have shown that there are two equally likely movements:

(i) forward in the $3^{\prime}$ direction on the road from STATE(i) to STATE(i+1), and

(ii) reverse in the $5^{\prime}$ direction on the road from STATE(i) to STATE(i-1).

Expected drift via random translational movement. We have shown that the resulting movement forms a random, bidirectional translational movements in both directions along the road. Due to the symmetry of the construction, both translational movements would have equal probability in either direction. It is well known (see Feller, 1971) from the theory of random walks in 1 dimension, that the expected deviation after $\mathrm{n}$ steps is $\mathrm{O}(\sqrt{n})$.

The infeasibility of latched movement by the walker fueled by heat energy. The above Walker DNA construction, can be viewed as executing a very simple type of autonomous motion, which is both translational and rotational, fueled by kinetic (heat) energy. We can easily modify the above design to include a "latching mechanism" that fixes the walker's position at specified locations along the road. This can be done by appending to each $3^{\prime}$ end of the walker an additional "latching" sequence and also inserting the complements of these "latching" sequences at a specified pair of locations along the road, which can fix (via their hybridization) the walker's location once the locations are reached and these "latching" hybridizations occur.

Hence at first it would appear that above Latched Walker DNA construction can realize latched movement just by use of Heat Energy. In fact, the latching of random movement is well known not to succeed: since total energy would not be conserved, it would violate the laws of thermodynamics. Hence by the laws of thermodynamics, this latched random autonomous motion will eventually reverse itself. The popular physics literature is full of schemes - violating the laws of thermodynamics, and so theoretically infeasible - for harnessing heat energy to fuel nanomechanical motion. Feynman in his Lectures on Physics (Feynman, 1969) gave a detailed critique of a proposed method (known as the Thermal Ratchet) for ratcheted movement using heat energy. 
However, Astumian $(1997,2001)$ discusses how latched or ratcheted molecular movement is feasible in many cases (and used by many biological motors) if there is a source of external potential energy fueling the movement. It generally results in a Brownian movement with a biased drift in a given direction. An example of a well studied protein motor using this scheme is the movement of myosin along an actin filaments (Kitamura, Tokunaga, Iwane and Yanagida, 1999), and related methods for quantum ratcheted movement have also been investigated (Humphrey, Linke and Newbury, 2001). For a brief overview of the use of such methods for the design of molecular motors with latched molecular movement using an external energy source, see (Musser, 1999).

We next consider the challenge of fueling random movement via some external potential energy source.

\section{Our rolling DNA device}

It is an interesting question whether bidirectional autonomous motion can be achieved without use of DNA ligase or any restriction enzyme and also without changes in the environmental condition of the system. Here we answer the question positively. We give a description of a DNA device (see Figure 1b), that we call Rolling DNA, which achieves bidirectional autonomous motion, without use of DNA ligase or any restriction enzyme and also without changes in the environmental condition of the system. The motion is random and bidirectional, forming a random walk along a DNA strand.

Overview of our rolling DNA construction. Let $A_{0}, A_{1}, B_{0}, B_{1}$ each be distinct oglionucleotides of low annealing cross-affinity, consisting of L (L typically would be between 15 to 20 ) bases pairs, and let $\bar{A}_{0}, \bar{A}_{1}, \bar{B}_{0}, \bar{B}_{1}$ each be their respective complements. Let $a_{0}, a_{1}, b_{0}, b_{1}$ be oglionucleotides derived from $A_{0}, A_{1}, B_{0}, B_{1}$ by changing a small number of bases, so their hybridization affinity with $\bar{A}_{0}, \bar{A}_{1}, \bar{B}_{0}, \bar{B}_{1}$ respectively is considerably reduced.

For $\mathrm{i}=0$ and 1 , we denote a hybridization between $A_{i}$ and complementary sequence $\bar{A}_{i}$ (or between $B_{i}$ and complementary $\bar{B}_{1}$ ) a full hybridization, where as a hybridization between $a_{i}$ and $\bar{A}_{i}$ (or between $b_{i}$ and $\bar{A}_{1}^{R}$ ) will be called a weak hybridization. We assume that at the appropiate temperature, a weak hybridization will be able to displace a weak hybridization.

We now define (using descriptive names) some DNA strands that will be of use:

(i) The road (see Figure 10) consists of an ssDNA with $a_{0}, a_{1}, a_{0}, a_{1}, a_{0}, a_{1}$, ... in direction from $5^{\prime}$ to $3^{\prime}$, consisting of a large number of repetitions of the sequences $a_{0}, a_{1}$. 


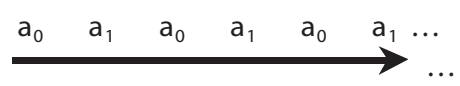

Figure 10. The DNA wheel's road.

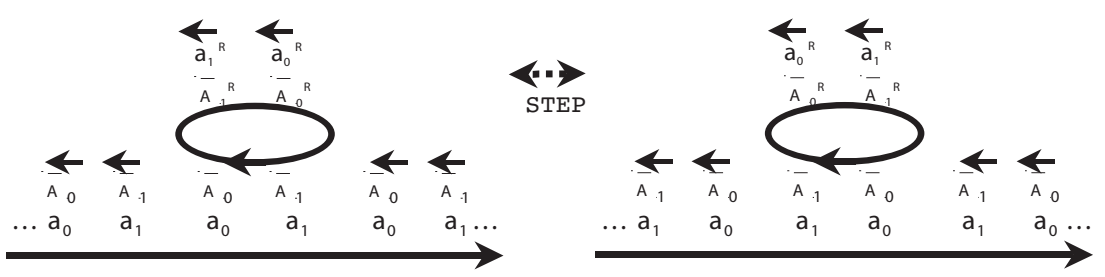

Figure 11. Type 0 and type 1 wheel positions.

(ii) The wheel caps consist of the oglionucleotides $a_{0}^{R}$ and $a_{1}^{R}$ which are the reverses of $a_{0}$ and $a_{1}$ as defined above. These wheel caps will be in solution and they will also weakly hybridize with each of the wheel oglionucleotides $\bar{A}_{0}^{R}$ and $\bar{A}_{1}^{R}$ respectively, except where displaced by various oglionucleotides as described below.

(iii) The wheel consists of a cyclic ssDNA of base length $4 \mathrm{~L}$ with $\bar{A}_{0}^{R}, \bar{A}_{1}^{R}$, $\bar{A}_{0}^{R}, \bar{A}_{1}^{R}$ in direction from $5^{\prime}$ to $3^{\prime}$; this corresponds to $\bar{A}_{1}, \bar{A}_{0}, \bar{A}_{1}, \bar{A}_{0}$ in direction from $3^{\prime}$ to $5^{\prime}$. (Note: in the following we will illustrate the wheel with the subsequences at the top of the wheel labeled in the $5^{\prime}$ to $3^{\prime}$ direction, and the subsequences at the bottom of the wheel labeled in the $3^{\prime}$ to $5^{\prime}$ direction.)

(iv) The road caps consist of the oglionucleotides $\bar{A}_{0}$ and $\bar{A}_{1}$ as defined above. These road caps will be in solution and they will also weakly hybridize with each of the road oglionucleotides $a_{0}$ and $a_{1}$ respectively, except where displaced by various oglionucleotides as described below.

The wheel has two distinguished positions with respect to the road:

(0) A type 0 wheel position, as illustrated on the left portion of Figure 11, where two of the wheel subsequences $\bar{A}_{0}, \bar{A}_{1}$ are weakly hybridized to a consecutive pair of subsequences $a_{0}, a_{1}$ on the road. (Note that on the left portion of Figure 11 lists $\bar{A}_{1}^{R}, \bar{A}_{0}^{R}$ at top of the circular DNA wheel in the $5^{\prime}$ to $3^{\prime}$ direction, where as the second pair below the wheel is written in the opposite $3^{\prime}$ to $5^{\prime}$ direction as $\bar{A}_{0}, \bar{A}_{1}$. Also, the subsequences $\bar{A}_{1}^{R}$, $\bar{A}_{0}^{R}$ at the top of the wheel are hybridized to the reverse of the wheel caps $A_{1}^{R}, A_{0}^{R}$.)

(1) A type 1 wheel position, as illustrated on the right portion of Figure 11, where two of the wheel subsequences $\bar{A}_{1}, \bar{A}_{0}$ are weakly hybridized to a consecutive pair of subsequences $a_{1}, a_{0}$ on the road. 
(Note again that the right portion of Figure 11 lists $\bar{A}_{0}^{R}, \bar{A}_{1}^{R}$ at top of the circular DNA wheel in the $5^{\prime}$ to $3^{\prime}$ direction, where as the second pair below the wheel is written in the opposite $3^{\prime}$ to $5^{\prime}$ direction as $\bar{A}_{1}, \bar{A}_{0}$. Also, the subsequences $\bar{A}_{0}^{R}, \bar{A}_{1}^{R}$ at the top of the wheel are hybridized with the wheel caps $a_{0}^{R}, a_{1}^{R}$.)

In each of these positions of the wheel, the road caps $\bar{A}_{0}$ and $\bar{A}_{1}$ will be weakly hybridized with the road oglionucleotides $a_{0}$ and $a_{1}$ respectively, except where displaced by segments of the wheel that hybridize with the road. Furthermore, wheel caps $a_{0}^{R}$ and $a_{1}^{R}$ will be weakly hybridized with the upper wheel oglionucleotides $\bar{A}_{0}^{R}$ and $\bar{A}_{1}^{R}$, respectively. However, in transitions between these type 0 and type 1 wheel positions, certain road caps and wheel caps will be displaced by various oglionucleotides (e.g., portions of fuel strands and road oglionucleotides) as described below. Road caps and wheel caps in solution will re-hybridize onto the road and wheel where the appropriate binding sites appear at the end of these transitions between wheel positions, as described below.

Random translational movement fueled by heat energy. Note that the kinetics (similar to those occurring in branch migrations classically found in Holiday junctions with exact symmetry, and very well understood), will allow for a slow rate of random movements of the wheel in direction $5^{\prime}$ to $3^{\prime}$ or its reverse, resulting in transitions between these two position types. Note that these translational movements would be in both directions along the road. Due to the symmetry of the construction, both translational movements would have equal probability in either direction. As noted in the previous section, the expected deviation after $n$ steps is $O(\sqrt{n})$.

Accelerated random movement fueled by DNA hybridization. Our construction, further detailed below, will provide for an acceleration of this random walk movement, by the use of fuel DNA strands, which provide a facility of energy exchange. This initial conformation of the fuel DNA is a state, which temporarily traps its hybridization energy. We will describe the application of DNA catalyst techniques for liberating DNA from these loop conformations, and harness their energy as they make transitions into lower energy conformations.

We wish to emphasize that the idea of using fuel DNA strands and hybridization catalysts to generate a sequence of motions is not a new idea; it was used by Yurke and Turberfield (Yurke, Turberfield, Mills, Simmel and Neumann, 2000) to demonstrate a series of DNA nanomechanical devices such as their DNA tweezers. However, to induce repetitions of the motions 


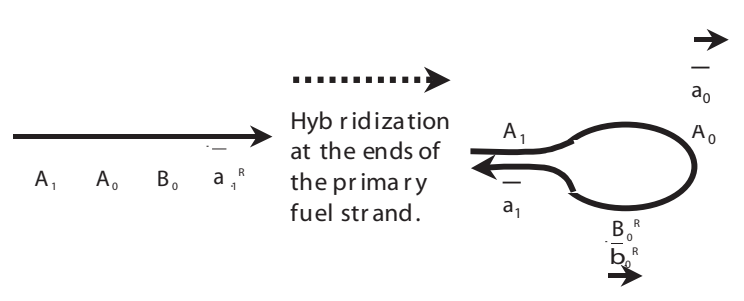

Figure 12. The type 0 primary fuel strand.

by their DNA devices, their devices required external environmental changes; in particular, the use of biotin-streptavidin beads.

Our main contibution here beyond that prior work is that here we require no external environmental changes to induce repetitions of the motions by our DNA devices. Instead, we will gradually consume the energy of the DNA fuel loop strands. (This was suggested to us by Turberfield as a possible mechanism for obtaining autonomous motion.)

Details of our device construction. (Note: in the following we will illustrate the fuel strands with the subsequences at the top of the stem loops labeled in the $5^{\prime}$ to $3^{\prime}$ direction, and the subsequences at the bottom of the stem loops labeled in the $3^{\prime}$ to $5^{\prime}$ direction.) We define: the type 0 primary fuel strand, as illustrated on the left portion of Figure 12, consisting of an ssDNA of base length $4 \mathrm{~L}$ with $A_{1}, A_{0}, B_{0}, \bar{a}_{1}^{R}$. Initially, we assume an initial loop conformation (prepared in a distinct test tube from the complementary fuel strand) of the type 0 primary fuel strand, as illustrated on the right portion of Figure 12, with its $5^{\prime}$ end segment $A_{1}$ weakly hybridized to its $3^{\prime}$ end segment $\bar{a}_{1}^{R}$. Also in this initial loop conformation, its second segment will be $A_{0}$ weakly hybridized to an additional strand $\bar{a}_{0}$, and with its third segment $B_{0}$ weakly hybridized to an additional strand $\bar{b}_{0}$ (in this context we call $\bar{a}_{0}, \bar{b}_{0}$ the primary fuel strand's protection segments).

A type 0 complementary fuel strand, as illustrated on the left portion of Figure 13, consists of an ssDNA of base length $4 \mathrm{~L}$ with $a_{1}, \bar{B}_{0}^{R}, \bar{A}_{0}^{R}, \bar{A}_{1}^{R}$. Similarly, we assume the following initial loop conformation of the type 0 complementary fuel strand, as illustrated on the right portion of Figure 13, with its $5^{\prime}$ end segment $\bar{a}_{1}$ weakly hybridized to its last segment $A_{1}^{R}$, its second segment $\bar{B}_{0}^{R}$ weakly hybridized to an additional strand $b_{0}^{R}$, and its third segment $\bar{A}_{0}^{R}$ is weakly hybridized to an additional strand $a_{0}^{R}$ (in this context we call $b_{0}^{R}, a_{0}^{R}$ the complementary fuel strand's protection segments). (The type 1 primary fuel strands and type 1 complementary fuel strands are defined identically to the type 0 primary and complementary fuel strands, respectively, but with the subscripts 0 and 1 exchanged.) 


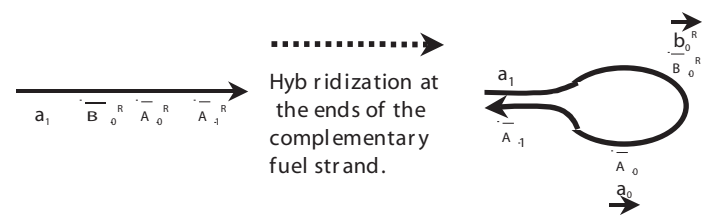

Figure 13. The type 0 complementary fuel strand.

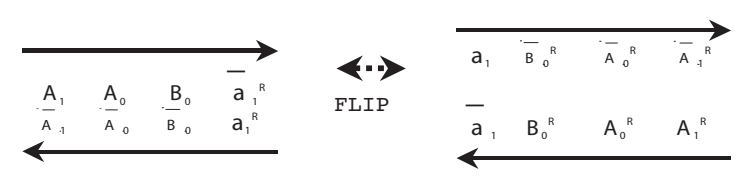

Figure 14. A duplex resulting from full hybridization of the DNA fuel strand with the wheel, and a flip of the duplex.

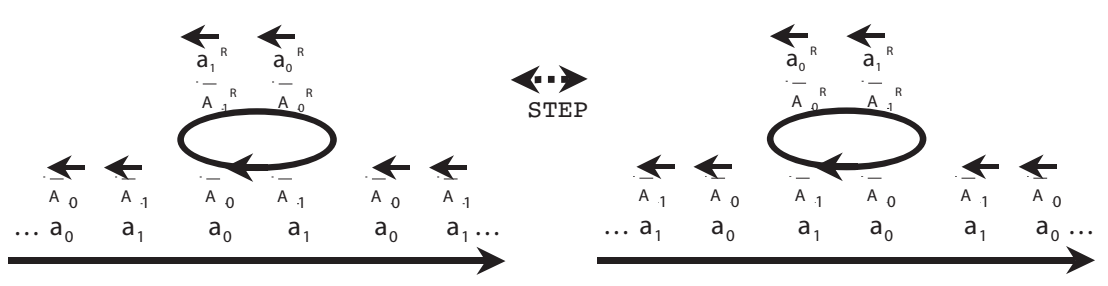

Figure 15. One step of the wheel's movement.

The sequence of events of a feasible movement. Now suppose the wheel is in type 0 position with respect to the road as illustrated on the left of Figure 15. Observe that the $0^{\text {th }}$ primary fuel strand can interact with the wheel in the type 0 position by the following hybridizations:

(i) First a hybridization of the second segment $A_{0}$ of the $0^{\text {th }}$ primary fuel strand with the reverse complementary segment $\bar{A}_{0}^{R}$ of top of the wheel results in the conformation illustrated on the middle portion of Figure 16.

(ii) Then, as illustrated in the middle to the right portions of Figure 16, that single segment hybridization extends to a hybridization of two first segments $A_{1}, A_{0}$ (in the $3^{\prime}$ to $5^{\prime}$ direction) of the $0^{\text {th }}$ primary fuel strand with the consecutive reverse complementary segments $\bar{A}_{1}^{R} \bar{A}_{0}^{R}$ (in the $5^{\prime}$ to $3^{\prime}$ direction) of the wheel, so the wheel moves by one segment in the $5^{\prime}$ direction along the road, effecting a transition of the state of the wheel from the type 0 position to type 1 position. This results in the conformation illustrated on the right portion of Figure 16.

(iii) Next, as illustrated in the middle portion of Figure 17, a type 0 complementary fuel strand consisting of an ssDNA with $\bar{A}_{1}, \bar{B}_{0}^{R}, \bar{A}_{0}^{R}, A_{1}^{R}$ (in the $3^{\prime}$ to $5^{\prime}$ direction) is now susceptible to hybridizations with reverse 


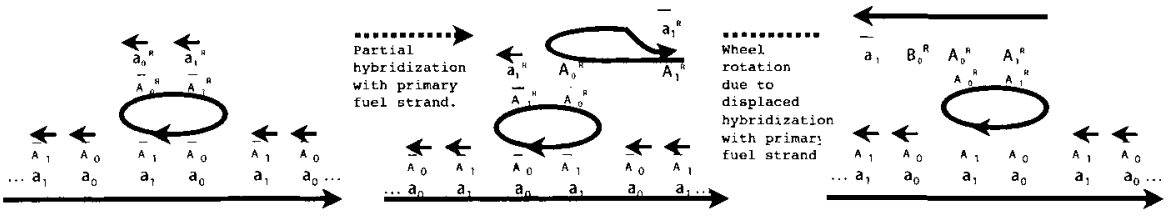

Figure 16. Rotation of the DNA wheel induced by partial hybridization with a fuel strand.

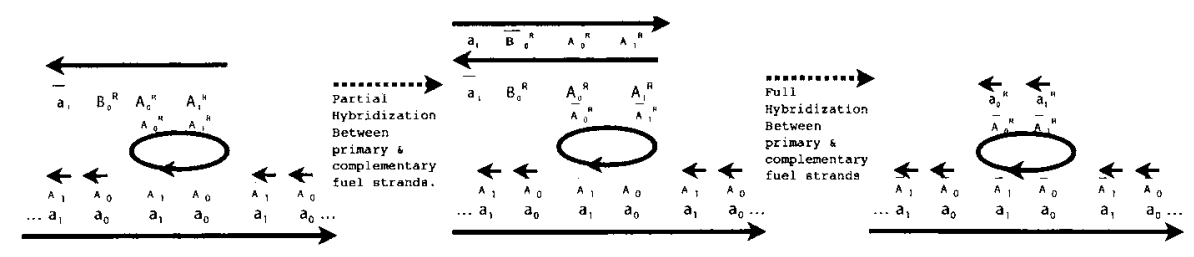

Figure 17. Full hybridization of paired fuel strands which depart from the wheel, completing the step.

complementary subsequences of the type 0 primary fuel strand, first at that fuel strand's newly exposed $3^{\prime}$ end segment $A_{1}^{R}$ and then at $B_{0}$. The resulting type 0 fuel strand duplex consists of the mutual hybridization of the entire type 0 primary fuel strand with the type 0 complementary fuel strand, as illustrated on the right portion of Figure 14. (Note: The same resulting duplex of the type 0 fuel strands is also illustrated on the left portion of Figure 14 - it is flipped top to bottom and left to right, as also described in Figure 2 - so it can be compared with the prior illustrations of the type 0 primary fuel strands.) The formation of this type 0 fuel strand duplex removes the type 0 fuel strands from the wheel.

Also, at the completion of the step, the subsequences $\bar{A}_{0}^{R}, \bar{A}_{1}^{R}$ at the top of the wheel will be hybridized to wheel caps $a_{0}^{R}$ and $a_{1}^{R}$ respectively, from the solution. This results in the conformation illustrated on the right portion of Figure 17. Again, see the Discussion Section for a description of the use of various methods to avoid unwanted interactions.

Summary of the basic wheel movement. We conclude that:

(a) There is a feasible transition of the state of the wheel from type 1 position to type 0 position driven by the fuel strands of type 0 , where the wheel moves by one segment in the $5^{\prime}$ direction along the road.

(b) Also, there is also a similarly feasible transition of the state of the wheel from a type 0 position to type 1 position driven by the type 1 fuel strands, where again the wheel moves by one segment in the $5^{\prime}$ direction along the road. (This is exactly the same as described above, but with 0 and 1 reversed.) 
Other feasible movements. The above does not ensure the movement is only in the $5^{\prime}$ direction along the road. In fact, there are multiple other feasible interactions of the wheel with the fuel strands. By a similar argument, it can easily be shown:

(c) There is a feasible transition of the state of the wheel from type 1 position to type 0 position driven by the type 0 fuel strands, where the wheel moves by one segment in the $3^{\prime}$ direction along the road.

(d) There is a feasible transition of the state of the wheel from a type 0 position of to a type 1 position driven by the type 1 fuel strands, where the wheel moves by one segment in the $3^{\prime}$ direction along the road.

(e) For each $\mathrm{i}=0,1$, there is a feasible hybridization of the exposed (top) portion of the wheel in a type i position with a fuel strand that makes no transition of the state of the wheel (the wheel remains in a type i position, though the fuel strand is transformed into its duplex state), so the wheel stalls for a moment in its movement along the road.

Random bidirectional movement of the wheel. Hence the movement of the wheel with respect to the road remains a random walk with either direction or a momentary stall possible on each step with fixed probability. By symmetry in the construction, it can be shown that the likelihood of each direction (from $5^{\prime}$ to $3^{\prime}$, or its reverse) of movement is equally likely. Recall again that for unbiased random walks in 1 dimension, that the expected translational deviation after $\mathrm{n}$ steps is $\mathrm{O}(\sqrt{n})$. However, the fuel strands appear to considerably accelerate the rate that steps of this random walk are executed (as compared to random movements fueled by kinetic (heat) motion). Hence the acceleration in the rate of these transitions may be of use, and the above has the potential of being feasible design of an autonomous bidirectional DNA nanomechanical device without use of DNA ligase or restriction enzymes.

A latched DNA wheel construction for overall unidirectional translational movement. We can modify the above DNA Wheel design to include a "latching mechanism" that fixes the wheels position at specified locations along the road (e.g., by hybridization with an additional pair of complementary ssDNA strands inserted into in the wheel and also at a specified pair of locations along the road, which can fix the wheel's location once the locations are reached and these annealing occur). Even though each step until latching unidirectional, the overall movement after latching is a unidirectional translation. Note that in each such step of this translational movement, the wheel will simultaneously rotate around its center by $2 \pi / 4=$ $\pi / 2$ radians. Again, due to the well-known secondary structure of B-form dsDNA (rotating $2 \Pi$ radians every approximately 10.5 bases), it follows 
that the wheel will also rotate around the axis of the road by approximately $L 2 \pi / 10.5=L \pi / 5.25$ radians. (Note: This Latched DNA Wheel construction for realizing overall unidirectional translational movement does not violate the laws of thermodynamics since its energy source is the DNA hybridization potential energy from the fuel DNA added to the system, as described by Astmian $(1997,2001)$ and Musser (1999).)

\section{Discussion}

Avoiding unwanted interactions. We give here a brief description of various methods (loosely immobilizing the road suggested settings of road and walker concentrations) that may be used to avoid unwanted interactions.

To ensure there is no interaction between a wheel (or walker) and more than one distinct road at a time, we use a sufficiently low absolute road concentration and we also loosely immobilize the road. To ensure there is no interaction between a road and more than one wheel (or walker), we use a sufficiently low wheel (or walker) concentration relative to the determined road concentration.

To loosely immobilize the road, we can use AFM manipulation (or possibly water flow) without requiring solid support attachment of the road. This will insure the road is laid out so that the walker is limited to adjacent sites only.

This use of a loosely immobilized road and sufficiently long walker segments should also insure that the enzymes can work properly on the walker. This use of a loosely immobilized road and sufficiently long walker segments should also insure that the enzymes can work properly on the walker.

(Solid support attachment of the road may be less practical, since it may disable enzymes activity near the surface attachment. Furthermore, solid support attachment requires a modification of the road using additional distinct subsequences to be used for attachment hybridizations of the road to the solid support.)

Energetics of the fuel strands: Analysis from the viewpoint of thermodynamics (Gibbs energy). To understand the energetics of the fuel strands, we will first briefly review the energetics of DNA hybridization. Hydrogen bonds have energy of between 3 and $5 \mathrm{Kcal} / \mathrm{mole}$. Recall that an hybridization between bases A,T has 2 hydrogen bonds whereas an hybridization of bases $\mathrm{G}, \mathrm{C}$ has 3 hydrogen bonds, so is a thermodynamically stronger bond. The dsDNA melting temperature $T_{m}$ increases 2 degrees per AT base pair and 4 degrees per GC base pair. 
If we choose $A_{0}, A_{1}, B_{0}, B_{1}$ to all have the same base length $\mathrm{L}$ between 15 to 20 bases, and require them (i.e., these sequences, their reverses, their complements, and their reverse complements) to differ by at least 4 bases in any possible binding configuration, then the melting temperature of incorrectly matched sequences will be at least 10 degrees. They will have very low annealing cross-affinity since the potential energy difference between correctly and incorrectly hybridized pairs is significant.

If we let $a_{0}, a_{1}, b_{0}, b_{1}$ be oglionucleotides derived from $A_{0}, A_{1}, B_{0}$, $B_{1}$ by changing 2 bases, their hybridization affinity with $\bar{A}_{0}, \bar{A}_{1}, \bar{A}_{0}, \bar{A}_{1}$ respectively will be considerably reduced since the potential energy on such weakly hybridized pairs $\left(a_{i}-\bar{A}_{i}\right.$, or $b_{i}-\bar{B}_{i}$, where $\left.i=0,1\right)$ is significantly reduced compared to the potential energy on fully hybridized pairs $\left(A_{i}-\bar{A}_{i}\right.$, or $A_{i}-\bar{B}_{i}$, where $i=0,1$.). Hence at the appropriate temperature a full hybridization will be able to displace a weak hybridization of the same length.

The initial conformations of the primary and secondary fuel strands (see Figures 12 and 13) have each three weak hybridizations (each on a segment of $\mathrm{L}$ bases). The duplex DNA of the type 0 primary and complementary fuel strands is as given in Figure 14; it has a complete hybridization of all four of their their component segments. (The duplex consisting of the complete hybridization of the type 1 primary and complementary fuel strands is identical, but with the 0 and 1 subscripts exchanged.) Since initial three weak hybridizations of each fuel strand are replaced by four full hybridizations, the net potential energy of these strands is significantly lowered from the initial to final conformations. The whole complementarity of the primary and complementary fuel strands of a given type implies that the duplex DNA resulting from their complete hybridization has lower free energy. So eventually, over a sufficiently long time interval, the free energy will drive these two species to a double stranded duplex, which is their lowest energy equilibrium state. (For example, the Seeman lab has previously demonstrated (Lu, Guo, Marky, Seeman and Kallenbach, 1992) that when two 4-arm junctions are mixed together at an appropriately high temperature, and cooled, they will go to a 4 duplex.)

We are concerned with the special case where the primary fuel strands and the complementary fuel strand are prepared separately to form the initial conformations define above. By setting a sufficiently low temperature, that equilibrium duplex state can be made to take any given time duration to reach on the average. So we will use an appropriately chosen sufficiently low temperature to ensure the stability of the initial state (prepared as given in Figures 12 and 13) of a fraction $\epsilon$ of the fuel strands for at least a few few minutes (i.e., this is the expected time duration where the primary and complementary fuel strands hold their initial state and do not anneal to each 
other to form a duplex state). Due to the linear rate of degradation of the fuel strands, $O(1 / \epsilon)$ cycles of motions described below will then be feasible within this limited time interval.

The kinetics of hybridization cooperation. Cooperation can play a major role in the kinetics of DNA hybridization events. For example, if a ssDNA $X$ of length $\mathrm{L}$ hybridizes at segment $\bar{X}$ of a ssDNA $\overline{X Y}$ of length $2 \mathrm{~L}$ to form a partial duplex, and then a further ssDNA $X Y$ of length $2 \mathrm{~L}$ hybridizes at segment $\bar{Y}$ of the same ssDNA $\overline{X Y}$, then $X Y$ will tend to displace the ssDNA $X$, to form a full duplex hybridization with $\overline{X Y}$. The ssDNA $X$, once displaced will tend to drift away, where as the full duplex will be very stable.

Therefore in the analysis of hybridization cooperation, it is critical to examine the final full duplex's energy versus the energy of the initial partial duplex, but the energy of the ssDNA $X$, once it has drift away, is not very critical to the relevant subsequent kinetics as they concern the final full duplex.

Yurke and Turberfield (Yurke, Turberfield, Mills, Simmel and Neumann, 2000) also used this technique of DNA hybridization and displacement for their DNA tweezers. We will also exploit such cooperative kinetics of DNA hybridization and displacement in our DNA wheel construction, as described below.

Energetics of the phases of the wheel movement. In phase (i), recall that the hybridization of the second segment $A_{0}$ of the $0^{\text {th }}$ primary fuel strand with the reverse complementary segment $\bar{A}_{0}^{R}$ displaces a prior weak hybridization of the $0^{\text {th }}$ primary fuel strand's second segment $A_{0}$ with it's protection segment $\bar{a}_{0}$. The prior weak hybridization of segment $\bar{A}_{0}^{R}$ of the top of wheel strand with wheel cap $a_{0}^{R}$ will also be displaced by the full hybridization of the second segment $A_{0}$ of the $0^{\text {th }}$ primary fuel strand with the reverse complementary segment $\bar{A}_{0}^{R}$ of the wheel. Hence both the wheel and the primary fuel strand have a weak hybridization of length $L$ replaced by a full hybridization of length L, so each of their net potential energy is significantly lowered. Furthermore, the road's net potential energy is conserved.

In phase (ii) recall that the single segment hybridization extends to a hybridization of two first segments $A_{1}, A_{0}$ of the wheel. In this transition, the previous weak hybridization of these wheel subsequences with subsequences of the road will be displaced by the full hybridization of subsequences of the primary fuel strand with the reverse complementary segments of the wheel, so the wheel moves by one segment in the $5^{\prime}$ direction along the road, effecting a transition of the state of the wheel from the type 0 position to type 1 position. In particular: 
(a) This displaces the prior weak hybridization of lower wheel segment $\bar{A}_{1}$ with the road segment $a_{1}$, but this change in energy is balanced by a weak hybridization of another wheel segment $\bar{A}_{1}$ with the road segment $a_{1}$.

(b) This also displaces the prior weak hybridization of road cap $\bar{A}_{1}$ with the road segment $a_{1}$ which was just to the left of the wheel, but this change in energy is balanced by a weak hybridization of another road cap $\bar{A}_{1}$ with the road segment $a_{1}$ now just to the right of the wheel.

(c) This further displaces the prior weak hybridization of the the primary fuel strand's $5^{\prime}$ end segment $A_{1}$ with its $3^{\prime}$ end segment $\bar{a}_{1}^{R}$ of (which now is exposed), but that has less energy than the new full hybridization of the new upper right wheel segment $\bar{A}_{1}^{R}$ with the road segment $a_{1}$.

Again both the wheel and the primary fuel strand have a weak hybridization of length $\mathrm{L}$ replaced by a full hybridization of length $\mathrm{L}$, so each of their net potential energy is significantly lowered. Again, the road's net potential energy is conserved.

In phase (iii) recall a type 0 complementary fuel strand consisting of an ssDNA of base length $4 \mathrm{~L}$ with $\bar{A}_{1}, \bar{B}_{0}^{R}, \bar{A}_{0}^{R}, A_{1}^{R}$ is now susceptible to hybridizations with reverse complementary subsequences of the type 0 primary fuel strand, first at that fuel strand's newly exposed $3^{\prime}$ end segment $A_{1}^{R}$ and then at $B_{0}$. This also will displace an initial weak hybridization of the third segment $B_{0}$ of the $0^{\text {th }}$ primary fuel strand with the protection segment $\bar{b}_{0}$ (which for simplicity is not indicated in on the left portion of Figure 17). It also displaces two prior full hybridizations of the first and second segments $A_{0}^{R}, A_{1}^{R}$ of the $0^{\text {th }}$ complementary fuel strand with the top segments $\bar{A}_{0}^{R}, \bar{A}_{1}^{R}$ of the wheel.

Observe that the wheel has two full hybridizations replaced by two weak hybridizations, so it's net potential energy is increased. However the complementary fuel strand has three weak hybridizations of length $\mathrm{L}$ replaced by four full hybridizations of length $\mathrm{L}$ and also the primary fuel strand has one weak hybridization of length $\mathrm{L}$ replaced by two additional full hybridizations of length L, so both their net potential energy is lowered. Again, the road's net potential energy is conserved. Summing these energy changes, we see that the net potential energy is significantly lowered, since a total of six weak hybridizations and two full hybridizations are replaced by two weak hybridizations of length $\mathrm{L}$ and six full hybridizations of length $\mathrm{L}$.

Open problems. Key remaining challenges are to design a DNA device where each step is unidirectional and to experimentally demonstrate a DNA device achieving unidirectional movement (either translational or rotational). 


\section{Acknowledgments}

We wish to thank Tingting Jiang, Thom Labean, Nadrian Seeman, Andrew Turberfield, Hao Yan, and Peng Yin as well as the referees for their valuable and insightful comments that led to many improvements in this paper. As stated above, Turberfield proposed to us the use of DNA fuel loop strands to provide energy for autonomous motion by DNA devices and some possible mechanisms to use them, but these were distinct from what we have presented.

Supported by DARPA/AFSOR F30602-01-2-0561, NSF ITR EIA0086015, NSF EIA-0218376, and NSF EIA-0218359. Paper URL: http:// www.cs.duke.edu/ reif/paper/DNAmotor/DNAmotor.pdf.

\section{References}

Astumian RD (1997) Therodynamics and kinetics of a brownian motor. Science 276: 917-922 Astumian RD (2001) Making molecules into motors. Scientific American 285: 57-64

Benenson Y, Paz-Elizur T, Adar R, Keinan E, Livneh Z and Shapiro E (2001) Programmable and autonomous computing machine made of biomolecules. Nature 414: 430-434

Feller W (1971) An Introduction to Probability Theory and Its Applications. John Wiley \& Sons, New York

Feynman R (1969) Feynman Lectures on Physics, Vol I, Ch. 46. The Thermal Ratchet, Addison-Wesley Publishing, Reading, MA

Humphrey T, Linke H and Newbury R (2001) Pumping Heat with Quantum Ratchets, to appear in Physica E 11/2-3, 281 (2001), cond-mat/0103552

Kitamura K, Tokunaga M, Iwane AH and Yanagida T (1999) A single myosin head moves along an actin filament with regular steps of 5.3 nanometers. Nature 397: 129-134

LaBean TH, Yan H, Kopatsch J, Liu F, Winfree E, Reif JH and Seeman NC (2000) Construction, analysis, ligation, and self-assembly of DNA triple crossover complexes. J. Am. Chem. Soc. 122: 1848-1860

LaBean TH, Winfree E and Reif JH (2000) Experimental progress in computation by selfassembly of DNA tilings, Gehani, A. T.H. LaBean and J.H. Reif. In: Winfree E and Gifford DK (eds) DNA-based Cryptography, Proc. DNA Based Computers V: Cambridge, MA, June 14-16, 1999. DIMACS Volume 54, pp. 123-140. American Mathematical Society, Providence, RI. URL: http://www.cs.duke.edu/ reif/paper/DNAtiling/tilings/labean.pdf

Lu M, Guo Q, Marky LA, Seeman NC and Kallenbach NR (1992) Thermodynamics of DNA chain branching. Journal of Molecular Biology 223: 781-789

Musser G (1999) Taming Maxwell's Demon: Random molecular motions can be put to good use. Scientific American

Mao C, LaBean T, Reif JH and Seeman NC (2000) Logical Computation using algorithmic self-assembly of DNA triple crossover molecules. Nature 407: 493-496

Mao C, Sun W, Shen Z and Seeman NC (1999) A DNA nanomechanical device based on the B-Z transition. Nature 397: 144-146

Reif JH, LaBean TH and Seeman NC (2001) Challenges and applications for self-assembled DNA nanostructures. In: Condon A and Rozenberg G (eds) Proc. Sixth Inter.1 Workshop on DNA-Based Computers, Leiden, The Neth., June, 2000. DIMACS Lecture 
Notes in CS, Springer-Verlag, Berlin Heidelberg, vol. 2054, pp. 173-198. URL: http:// www.cs.duke.edu/ reif/paper/SELFASSEMBLE/selfassemble.pdf

Reif JH (2001) DNA lattices: A programmable method for molecular scale patterning and computation, to appear in the special issue on Bio-Computation, Computer and Scientific Engineering Journal of the Computer Society. URL: http://www.cs.duke.edu/ reif/ paper/DNAlattice/DNAlattice.pdf

Seeman NC (1999) DNA engineering and its application to nanotechnology, Trends in Biotech. 17: 437-443

Winfree FL, Wenzler LA and Seeman NC (1998) Design and self-assembly of twodimensional DNA crystals. Nature 394: 539-544

Yan H, Zhang X, Shen Z and Seeman NC (2002) A robust DNA mechanical device controlled by hybridization topology. Nature 415: 62-65

Yurke B, Turberfield AJ, Mills AP Jr, Simmel FC and Neumann JL (2000) A DNA-fuelled molecular machine made of DNA. Nature 406: 605-608 